\title{
Contralateral far-lateral approach for clipping of a ruptured vertebral artery-posterior inferior cerebellar artery aneurysm
}

\author{
Taryn McFadden Bragg, M.D., and Edward A. M. Duckworth, M.D. \\ Department of Neurological Surgery, Loyola University, Maywood, Illinois
}

\begin{abstract}
Numerous nuanced approaches have been used to access posterior inferior cerebellar artery (PICA) aneurysms for microsurgical clipping. The authors report the case of a patient with a right vertebral artery (VA)-PICA aneurysm that was reached via a contralateral far-lateral approach. The wide-necked saccular/fusiform aneurysm arose from the lateral aspect of the right $\mathrm{V}_{4}$ segment just proximal to the PICA origin, anterior to the jugular tubercle at the level of the hypoglossal canal. Computed tomography angiograms demonstrated the size and configuration of the aneurysm, and $3 \mathrm{D}$ reconstructions revealed the tortuosity of the right VA, defining its location just left of the midline adjacent to the lower clivus.

A contralateral far-lateral approach to VA-PICA aneurysms should be considered when aneurysms cross the midline. Computed tomography angiography with volume rendering and interactive software capabilities can help identify the relationship of such an aneurysm to an individual's particular skull base osseous anatomy and is paramount in selecting the optimal microsurgical approach. (DOI: 10.3171/FOC.2008.25.12.E9)
\end{abstract}

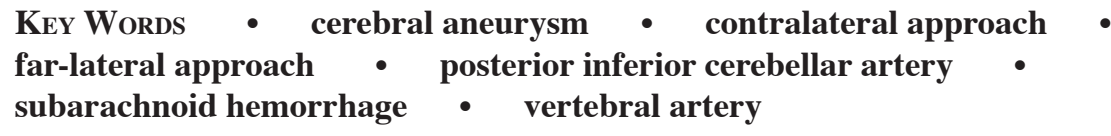

$\mathrm{A}$ NEURYSMS arising from the VA and PICA are rare, accounting for only $1.8-3 \%$ of all intracranial aneurysms. ${ }^{6}$ Two-thirds of all VA aneurysms, however, arise at or near the origin of the PICA. ${ }^{3}$ Surgical treatment of VA-PICA aneurysms can be difficult given the location of these aneurysms to the brainstem and lower cranial nerves. Various approaches, including specialized skull base approaches such as the lateral suboccipital, transcondylar, transcondylar fossa (supracondylar transjugular tubercle), extreme-lateral transcondylar, farlateral, and transpharyngeal-transclival have been described. ${ }^{4,5,10,11,13,15-17,19}$ With all aneurysms, factors such as size, morphology, and dome-to-neck ratio are critically evaluated before deciding the appropriate treatment modality (microsurgical or endovascular), as well as planning the operative approach. For aneurysms deep within the skull such as basilar trunk, vertebrobasilar junction, and many PICA aneurysms, other factors - such as osseous relationships and individual skull base anatomical nuances - can be just as critical. ${ }^{2}$ In the case of PICA aneurysms deemed appropriate for surgical intervention and located at the skull base, the anatomy of the jugular tubercle, hypoglossal canal, and all of the lower cra-

\footnotetext{
Abbreviations used in this paper: $\mathrm{CN}=$ cranial nerve; $\mathrm{PICA}=$ posterior inferior cerebellar artery; $\mathrm{SAH}=$ subarachnoid hemorrhage; $\mathrm{SCM}=$ sternocleidomastoid; VA = vertebral artery.
}

nial nerves must be considered.7 Computed tomography angiography can be very useful in preoperative assessment and surgical planning, especially when the studies are processed using interactive software, which can be manipulated to give realistic visual trajectories and expected intraoperative views. ${ }^{1,9,12,18}$ We report a case of a VA-PICA aneurysm located at the midline skull base, which was successfully clipped via a contralateral farlateral approach. Nuances of operative planning are discussed and the literature of this topic reviewed.

\section{Case Report}

Examination. This 54-year-old man presented with a 2-day history of headaches preceding a sudden change in mental status. His medical history was significant for untreated hypertension. A CT scan demonstrated diffuse thick SAH in the basal cisterns and in the prepontine and premedullary cisterns. Fourth ventricle blood was noted and there was evidence of acute hydrocephalus (Fig. 1). The SAH was classified as Hunt and Hess Grade IV on admission, but following ventricular drain placement, the patient's status improved such that he was opening his eyes and regarding and following commands symmetrically. Computed tomography angiography demonstrated a wide-necked saccular/fusiform aneurysm arising from the lateral aspect of the right $\mathrm{V}_{4}$ segment just proximal to the PICA origin. The aneurysm was located left of mid- 


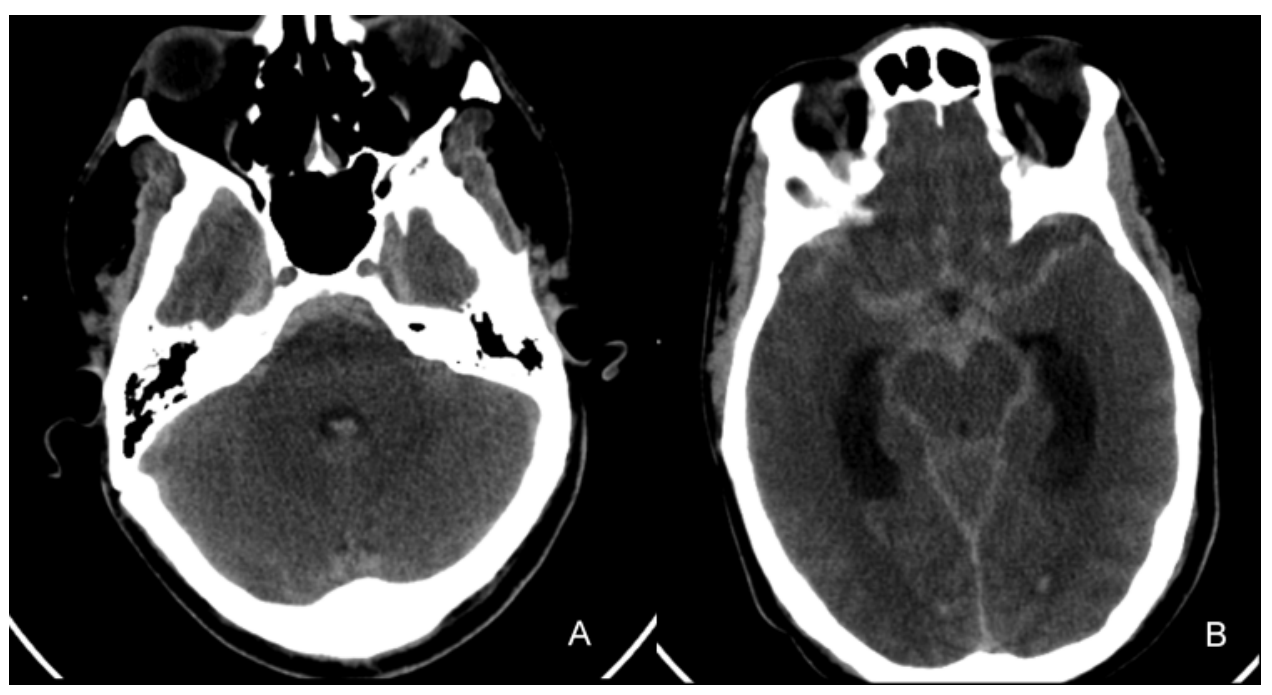

FIG. 1. Axial CT scans demonstrating, at admission, prepontine SAH (A) and diffuse cisternal SAH with acute hydrocephalus (B).

line, anterior to the jugular tubercle, at the level of the hypoglossal canal (Fig. 2).

Operation. Given the location of the artery and aneurysm, at the lower clivus just left of midline, a contralateral far-lateral approach was chosen. We believed an ipsilateral approach would not have provided the needed visual angles and operating trajectories necessary to access the aneurysm and, furthermore, that it would have exposed the dome of the aneurysm before the neck. A retrosigmoid approach would not have provided needed access anterior to the brainstem. Transclival approaches to this area have been described, ${ }^{8}$ but they require extensive, potentially destabilizing, bone drilling; involve traversing a nonsterile cavity; and require complex reconstructions to prevent postoperative cerebrospinal fluid leak.

The patient was placed in a three-quarter-prone position with the left side up. The head was placed in a radio-

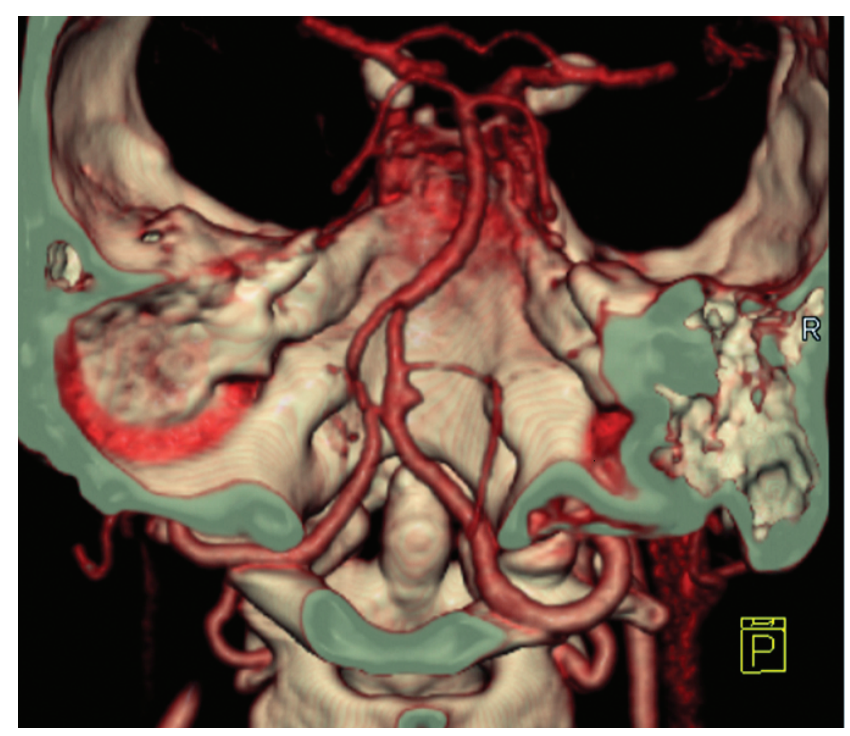

FIG. 2. Computed tomography angiogram demonstrating a right VAPICA aneurysm located to the left of the midline. lucent three-pin head holder with the head rotated slightly, flexed, and tilted away from the left shoulder (Fig. 3). A curvilinear retroauricular incision was made, extending into the upper cervical region along the posterior border of the SCM muscle (Fig. 4). The muscle layers, including the SCM, longisimus capitus, splenius and semispinalis capitus, and the suboccipital triangle muscles, were meticulously identified and separated. The SCM, longisimus, and superior and inferior oblique muscles were retracted anterolaterally, and the rest of the muscles were retracted posteromedially. The occipital artery was identified and preserved. The C-1 ring and lateral mass were identified along with the VA and its dural entry. A bur hole was made in the region of the asterion, and then a craniotome used to raise a small craniotomy flap. Some remaining bone at the foramen magnum was removed, and the occipital condyle was drilled to the level of the hypoglossal canal (Fig. 5). A curvilinear dural opening was made with a T-shaped incision toward the hypoglossal canal. Cranial nerves IX, X, and XI were identified and avoided. The CN XII rootlets were directly in the trajectory of our exposure and had to be manipulated somewhat during the procedure (Fig. 6). Despite manipulation, all of the rootlets remained anatomically intact. The ipsilateral PICA and VA were identified. Diffuse subarachnoid blood was evacuated. Following release of cerebrospinal fluid from the cisterna magna, the right VA was identified. As predicted on CT angiography, the aneurysm was pointing ventrally and to the right. The patient was placed in propofol-induced burst suppression and his body temperature allowed to cool to relative hypothermia $\left(36^{\circ} \mathrm{C}\right)$. Following arachnoid dissection, a temporary clip was placed on the right $\mathrm{V}_{4}$ segment. A $45^{\circ}$ fenestrated clip was placed on the aneurysm, and an intraoperative 4-vessel angiogram was obtained, which demonstrated persistent filling of the aneurysm. A second identical fenestrated clip was placed proximal to the first clip, and a second angiogram was obtained, this time revealing complete obliteration of the aneurysm and patent flow in the right VA and PICA (Fig. 7). The dura was reapproximated 


\section{Contralateral far-lateral approach for ruptured VA-PICA aneurysm}

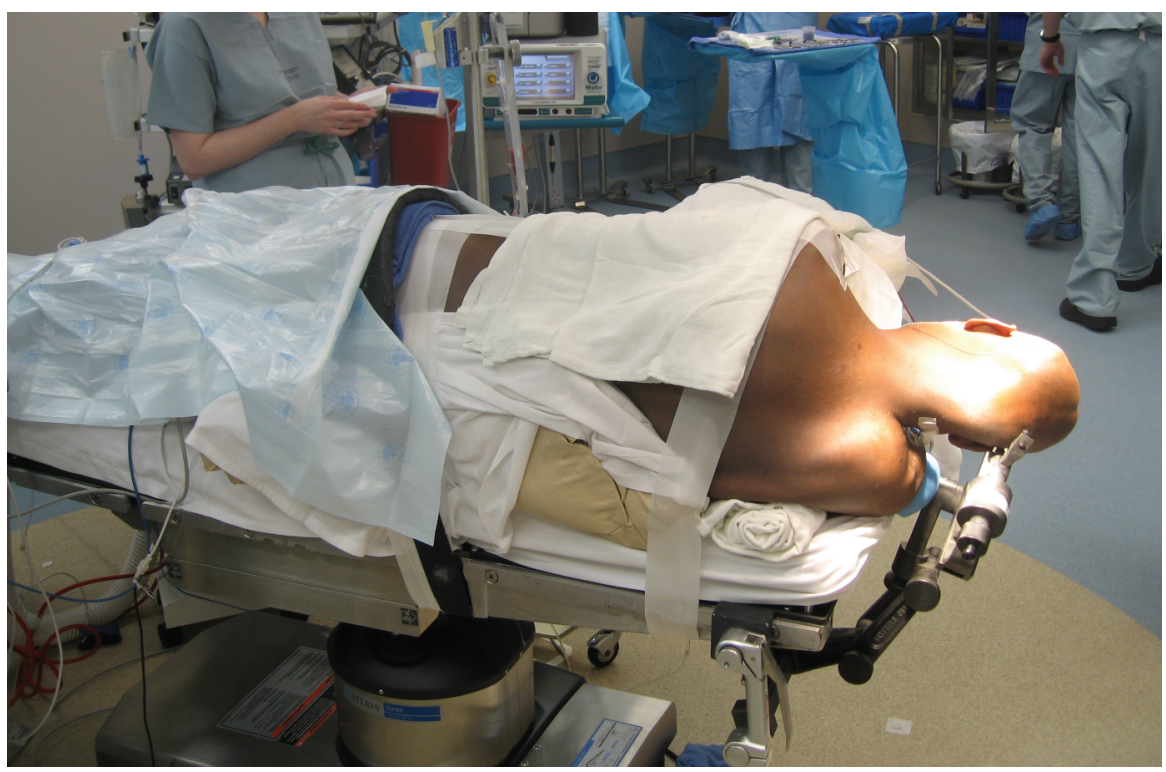

FIG. 3. The patient was placed in a three-quarter-prone position with his left side up. His head was positioned so as to optimize the far-lateral approach.

with Nurolon sutures (Ethicon), the defect remaining in the region of the condylar exposure covered with a small piece of Duragen (Integra Life Sciences), and the entire closure covered with a thin layer of DuraSeal (Confluent Surgical) to obtain a water-tight closure. The craniotomy bone flap was replaced and fixed in place with titanium mesh to cover the entire craniotomy defect. The muscles were methodically reattached to their insertion sites (Fig. 8 ), and the skin and scalp were then reapproximated in standard fashion.

Postoperative Course. Following extubation, the pa-

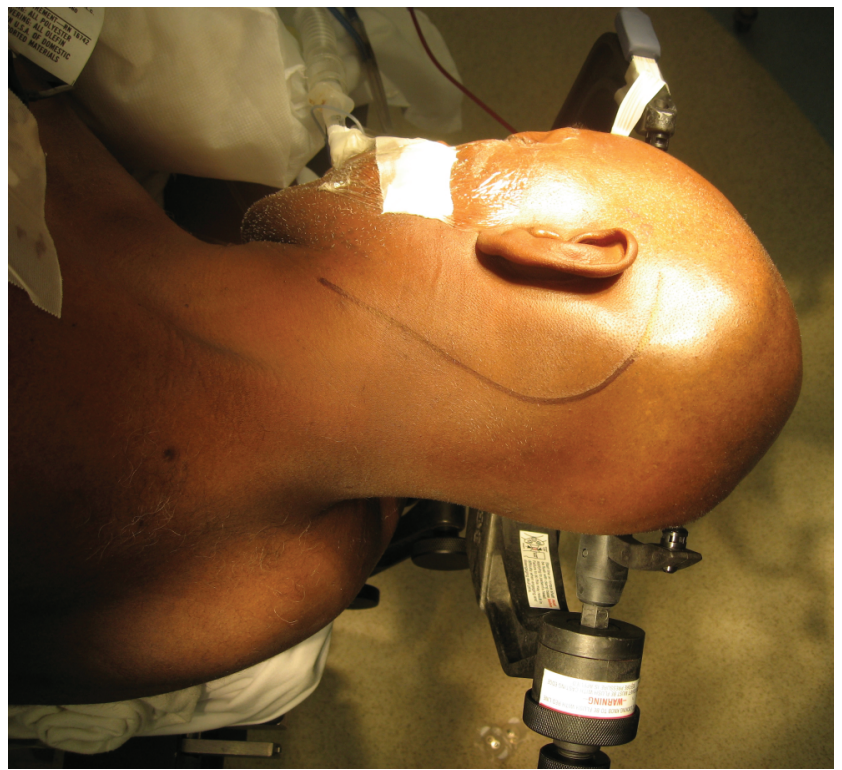

FIG. 4. A curvilinear retroauricular incision was marked, extending into the upper cervical region along the posterior border of the SCM muscle. tient was noted to have a left hypoglossal nerve palsy. His postoperative course was complicated by deep vein thrombosis, pulmonary embolus, and pneumonia requiring repeated reintubations. He underwent placement of a tracheostomy and a gastrostomy tube due to difficulty swallowing. The ventricular drain was weaned and discontinued without incident. The patient spent several weeks in acute rehab, but as of 2 months after surgery had his tracheostomy and gastrostomy tubes removed, was eating without difficulty, and was returning to work. His CN XII palsy has resolved, but the tongue remains somewhat atrophic.

\section{Discussion}

Aneurysms arising from the VA present unique surgi-

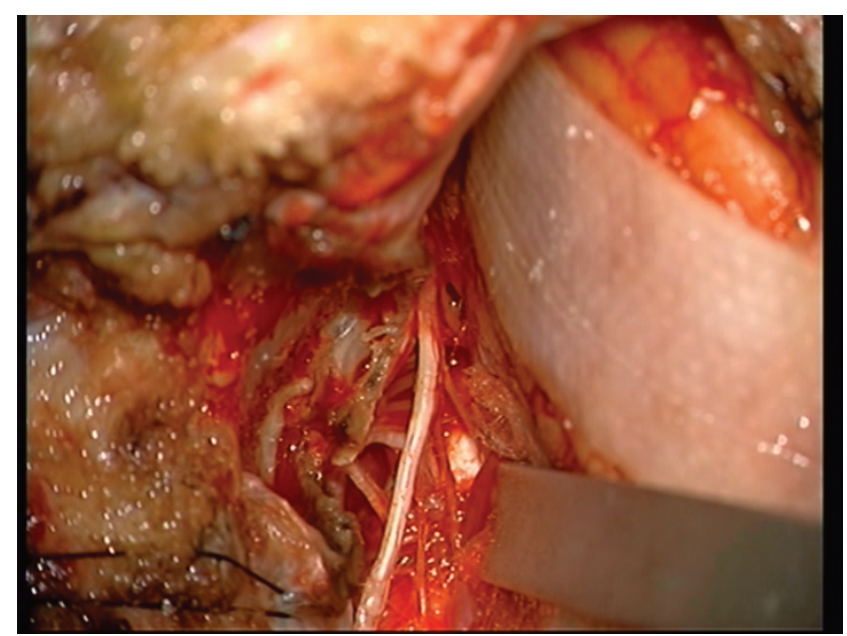

FIG. 5. Intraoperative microscopic image demonstrating the extent of occipital condylar drilling at the level of the hypoglossal canal. 


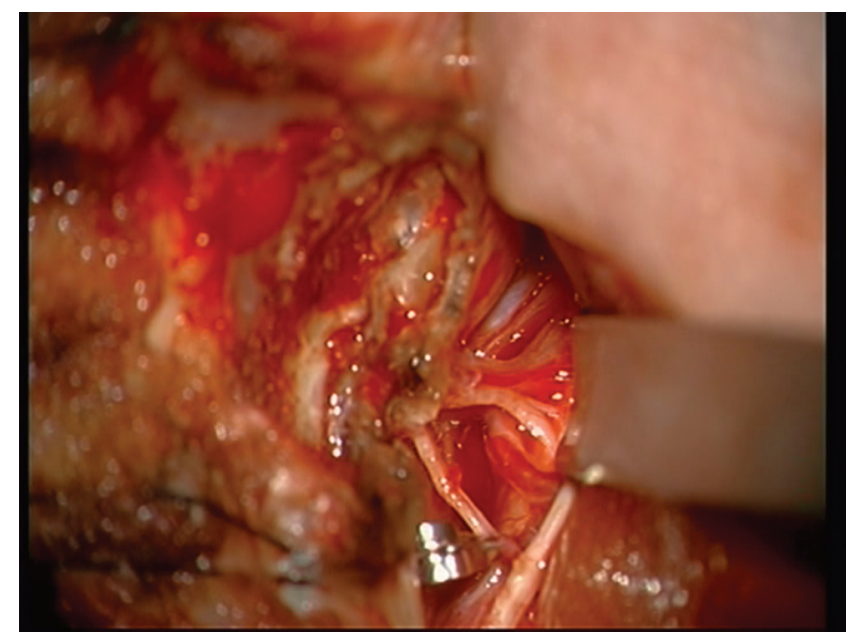

Fig. 6. Intraoperative image of the CN XII rootlets impeding aneurysm exposure. The $\mathrm{CN} \mathrm{XI}$ can be seen traversing the image vertically.

cal challenges given the complex osseous and neurovascular anatomy of the posterior fossa. Even when considering PICA aneurysms specifically, there is significant variability in the location and orientation of these lesions, ${ }^{17}$ which can be located near the dural entry of the VA (even extracranial), at the level of the foramen magnum, near the vertebrobasilar junction, midline, or contralateral to the midline. ${ }^{6,14}$ Furthermore, the tortuosity of the parent vessel can alter the aneurysm's relationship to the jugular tubercle, hypoglossal canal, and the cranial nerves. It is paramount that the characteristics of the aneurysm in relation to the anatomy of the foramen magnum be considered when planning the operative approach for an individual patient.

Computed tomography angiography with 3D reconstruction can be useful to identify key anatomical characteristics, such as aneurysm morphology, parent vessel

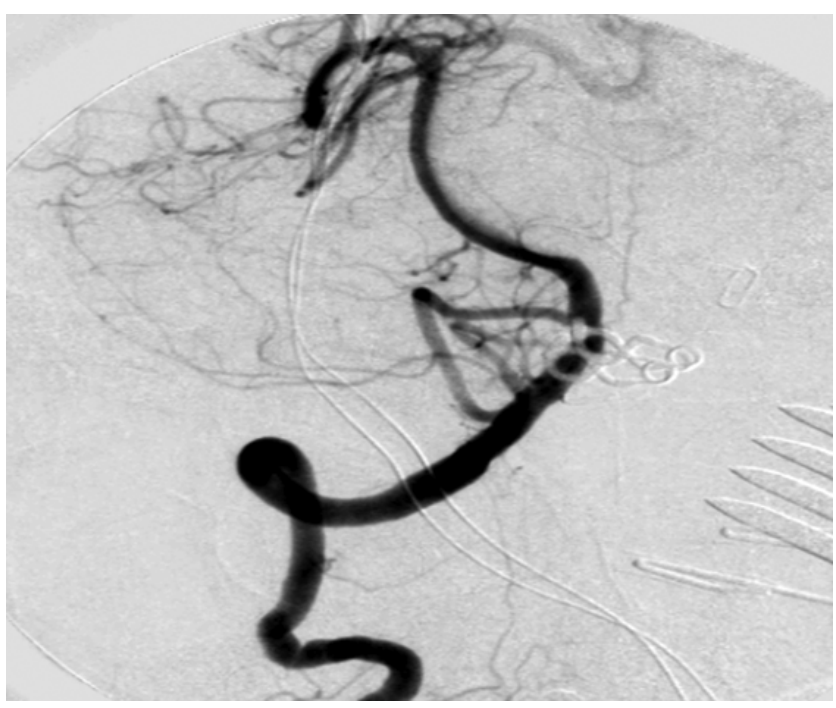

FIG. 7. Intraoperative cerebral angiogram demonstrating complete obliteration of the right PICA aneurysm using two $45^{\circ}$-angle fenestrated clips.

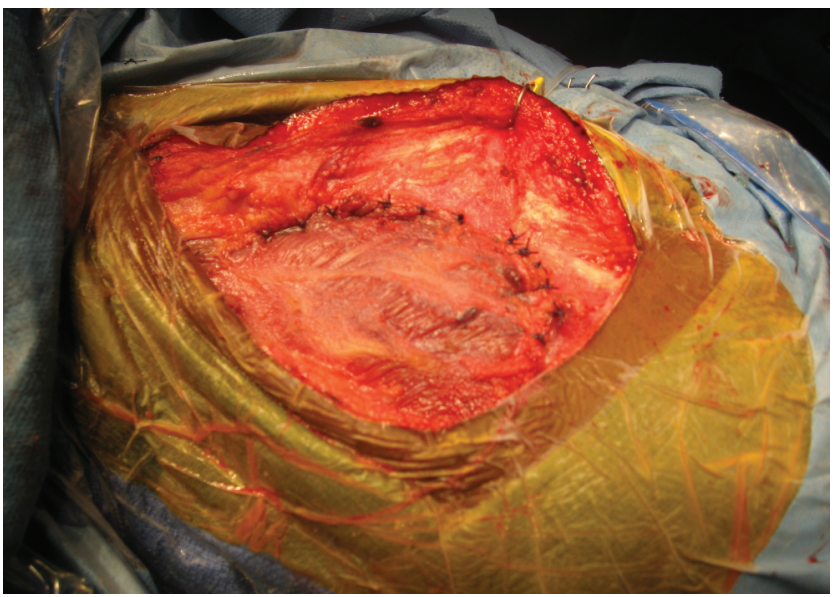

FIG. 8. Intraoperative photograph demonstrating anatomical reapproximation of the cervical muscle layers.

anatomy, and vascular-bony relationships, in cases like ours. ${ }^{1,4,9,12,18}$ Specifically, this imaging modality can be used to identify the relationship of a VA-PICA aneurysm to skull base landmarks such as the jugular tubercle, hypoglossal canal, and atlantooccipital joint. ${ }^{7}$ Furthermore, the orientation of the aneurysm with respect to the midline can be accurately assessed. When processed with software allowing interactive manipulation of the imaging data, 3D CT angiography enables the surgeon to rotate, zoom, and otherwise manipulate reconstructed images to help predict intraoperative anatomy ${ }^{7}$ and can help determine the nuances of skull base bone removal needed to optimize the visual trajectory.

Review of the literature provides numerous variations in the surgical approach to VA-PICA aneurysms, including the lateral suboccipital, transcondylar, transcondylar fossa (supracondylar transjugular tubercle), extremelateral transcondylar, far-lateral, and transpharyngealtransclival routes. ${ }^{2-6,10,11,13,15,16,19}$ There are, however, scant reports of contralateral approaches to VA-PICA aneurysms. The decision to use a contralateral far-lateral approach for our patient's aneurysm arising from the lateral (right) aspect of the right $\mathrm{V}_{4}$ segment just proximal to the PICA origin was based on the location of the artery and aneurysm to the left of midline and the aneurysm's orientation (the dome directed anteromedially with the aneurysm at the level of the hypoglossal canal ventral to the jugular tubercle). Taguchi et al. ${ }^{17}$ have reported a case of a left VA-PICA aneurysm treated successfully with a contralateral transcondylar approach, and Salcman et al. ${ }^{14}$ have described a case requiring a contralateral suboccipital approach. In both cases the authors noted that the decision to approach from the contralateral side was based on the tortuosity of the VA.

Recent trends note increased use of CT angiography as an adjunctive or sole diagnostic study of choice in the setting of suspected ruptured and unruptured aneurysms. The particular characteristics provided by CT angiography can be helpful in treatment decision making and surgical planning. The use of CT angiography in the present case enhanced our understanding of the surgical anatomy and significantly influenced the surgical approach. Al- 


\section{Contralateral far-lateral approach for ruptured VA-PICA aneurysm}

though indocyanine green video-angiography was used in our case, the information obtained was limited. This exciting, emerging technology depends on line of site visualization, which can be supremely difficult in the narrow confines of the posterior fossa. The orientation of the aneurysm relative to our approach necessitated the use of intraoperative catheter-based angiography, which provided invaluable information regarding complete obliteration of the aneurysm and patency of the afferent and efferent parent vessels.

\section{Conclusions}

Vertebral artery and PICA aneurysms can vary significantly in their relationship to the brainstem, cranial nerves, and osseous anatomy of the skull base of the posterior fossa. Surgeons should consider the use of a contralateral approach for certain aneurysms arising from ectatic VAs. We believe that CT angiography is invaluable in defining the anatomy of difficult aneurysms and should be considered for preoperative planning of such lesions.

\section{Disclaimer}

The authors report no conflict of interest concerning the materials or methods used in this study or the findings specified in this paper.

\section{References}

1. Carvi y Nievas MN, Haas E, Hollerhage HG, Drathen C: Complementary use of computed tomographic angiography in treatment planning for posterior fossa subarachnoid hemorrhage. Neurosurgery 50:1283-1289, 2002

2. Coert BA, Change SD, Do HM, Marks MP, Steinberg GK: Surgical and endovascular management of symptomatic posterior circulation fusiform aneurysms. J Neurosurg 106:855865, 2007

3. Drake CG: The treatment of aneurysms of the posterior circulation. Clin Neurosurg 26:96-144, 1979

4. Harbaugh RE, Schlusselberg DS, Jeffery R, Hayden S, Cromwell LD, Pluta D, et al: Three-dimensional computed tomographic angiography in the preoperative evaluation of cerebrovascular lesions. Neurosurgery 36:320-327, 1995

5. Heros RC: Lateral suboccipital approach for vertebral and vertebrobasilar artery lesions. J Neurosurg 64:559-561, 1986

6. Hudgins RJ, Day AL, Quisling RG, Rhoton Al Jr, Sypert GW, Garcia-Bengochea F: Aneurysms of the posterior inferior cerebellar artery. A clinical and anatomical analysis. J Neurosurg 58:381-387, 1983

7. Huynh-Le P, Matsushima T, Miyazono M, Sayama T, Muratani $\mathrm{H}$, Tashima T, et al: Three-dimensional CT angiography for the surgical management of the vertebral artery-posterior inferior cerebellar artery aneurysms. Acta Neurochir (Wein) 146:329-355, 2004.

8. Kassam AB, Gardner PA, Mintz A, Snyderman CH, Carrau RL, Horowitz M: Endoscopic endonasal clipping of an unsecured superior hypophyseal artery aneurysm. Technical note. J Neurosurg 107:1047-1052, 2007

9. Kato Y, Sano H, Katada K: Application of three-dimensional CT angiography (3D-CTA) to cerebral aneurysms. Surg Neurol 52:113-122, 1999

10. Kawase T, Bertalanffy H, Shiobara R, Otani M, Toya S, Shinozaki T, Ibata Y: Difference of surgical field between the lateral suboccipital approach and the transcondylar approach for the midline vertebral aneurysms. Surg Cereb Stroke 21:263-268, 1993

11. Matsushima T, Natori Y, Katsuta T, Ikezaki K, Fukui M, Rhoton AL: Microsurgical anatomy for lateral approaches to the foramen magnum with special reference to transcondylar fossa (supracondylar transjugular tubercle) approach. Skull Base Surg 8:119-125, 1998

12. Nakatsuka M, Mizuno S: Three-dimensional computed tomographic angiography in four patients with dissecting aneurysms of the vertebrobasilar system. Acta Neurochir (Wien) 142:995-1001, 2000

13. Rohde V, Schaller C, Hassler W: The extreme lateral transcondylar approach to aneurysms of the vertebrobasilar junction, the vertebral artery, and the posterior inferior cerebellar artery. Skull Base Surg 4:177-180, 1994

14. Salcman M, Rigamonti D, Numaguchi Y, Sadato N: Aneurysms of the posterior inferior cerebellar artery-vertebral artery comlex: variations on a theme. Neurosurgery 27:12-21, 1990

15. Sano K, Jimbo M, Saito L: Vertebro-basilar aneurysms with specific reference to the transpharyngeal-transclival approach to the basilar artery aneurysm. No To Shikei 18:1197-1203, 1966

16. Sen CN, Sekhar LN: An extreme lateral approach to intradural lesions of the cervical spine and foramen magnum. Neurosurgery 27:197-204, 1990

17. Taguchi Y, Hoshikawa Y, Tanaka K, Miyakita Y, Morishima $\mathrm{H}$, Sekino H: Contralateral transcondylar approach for aneurysms of the posterior inferior cerebellar artery-vertebral artery complex. J Clin Neurosci 3:156-161, 1996

18. Villavicencio AT, Gray L, Leveque JC, Fukushima T, Kureshi S, Friedman AH: Utility of three-dimensional computed tomographic angiography for assessment of relationships between the vertebrobasilar system and the cranial base. Neurosurgery 48:318-327, 2001

19. Wen HT, Rhoton AL Jr, Katsuta T, de Oliveira E: Microsurgical anatomy of the transcondylar, supracondylar, and paracondylar extensions of the far-lateral approach. J Neurosurg 87:555-585, 1997

Manuscript submitted August 16, 2008.

Accepted October 10, 2008.

Address correspondence to: Edward A. M. Duckworth, M.D., Department of Neurological Surgery, Loyola University, Maguire Building, Suite 1900, 2160 South First Avenue, Maywood, Illinois 60153. email: educkworth@lumc.edu.

\section{Comment by Dr. Vinko Dolenc}

This case report represents an interesting, well-designed, and appropriately executed exclusion of the aneurysm via the contralateral approach. The neurosurgeons dealing with vertebrobasilar system anomalies are well aware of the problem with issues of space when managing vascular lesions in the posterior cranial fossa. In addition to limitation of space in the VA-PICA aneurysm, CNs IX through XII lie in the path on the way to the target. Needless to say, any difficulties with placing the clip(s) on the aneurysm do result in weakness of one or more $\mathrm{CN}$ IX-XII, which might represent a very difficult problem in the postoperative care. This report is very welcome as it will enable us to consider the surgical strategy in similar situations as one of the approaches to the VA-PICA aneurysms. Due to the excellent advancement in endovascular treatment of the intracranial aneurysms and other patho- 
T. M. Bragg and E. A. M. Duckworth

logical entities, we have to be constantly advancing our surgical experience as well, because more and more patients are successfully treated with endovascular intervention. And when the direct surgical approach is considered as a better solution, also from the side of the endovascular specialist, then we are faced with challenging-but never simple and easy-cases. Only those surgeons who are mentally and physically prepared will be able to do the job. I do hope that neurosurgeons will push the cutting edge to the higher, safe, and reasonable level. 